\title{
Factors Related to Weight Gain in Subjects with Sleeve Gastrectomy During Lockdown by the COVID-19 Pandemic
}

\author{
Daniel de Luis ${ }^{1}$ (D) - Olatz Izaola ${ }^{1}$ - David Primo ${ }^{1}$ Emilia Gómez ${ }^{1}$ • Beatriz Torres ${ }^{1}$. Juan José López Gómez ${ }^{1}$. \\ David Pacheco ${ }^{1,2}$
}

Received: 10 December 2020 / Revised: 16 January 2021 / Accepted: 20 January 2021 / Published online: 1 February 2021

(C) The Author(s), under exclusive licence to Springer Science+Business Media, LLC part of Springer Nature 2021

\begin{abstract}
Purpose The COVID-19 pandemic could exacerbate the risk factors for weight gain in patients with previous bariatric surgery. The aim of this study was to evaluate factors related to weight gain during lockdown in patients with a sleeve gastrectomy. Materials and Methods A group of 48 obese subjects with previous bariatric surgery was enrolled. After a 7-week confinement, a telephone interview was conducted. In this phone call, self-reported body weight gain and different factors were recorded. In order to obtain the basal and pre-surgical data, biochemical and anthropometric parameters were recorded from electronic medical record.

Results The mean age was $45.3 \pm 8.0$ years (range: $23-61$ ) and the mean body mass index (BMI) was $32.5 \pm 7.5 \mathrm{~kg} / \mathrm{m}^{2}$ (range: 28.6-34.2). Gender distribution was 38 females (79.2\%) and 10 males (20.8\%). The increase in self-reported body weight was $3.8 \pm 2.1 \mathrm{~kg}$ during the 7 weeks of confinement. And the self-reported body weight gain was lower in subjects with regular exercise ( $4.6 \pm 0.9 \mathrm{vs} 1.1 \pm 0.3 \mathrm{~kg} ; p=0.02$ ). The number of face-to-face visits to the nutrition office that did not attend was 0.61 \pm 0.81 (range: $0-4$ ) per patient. In the multiple regression analysis with self-reported body weight gain as a dependent variable, the physical activity (minutes/week) remained as a protective factor with a beta coefficient of -0.09 (95\% CI: -0.001 to 0.016 ; $p=0.03$ ) and number of face-to-face appointments in the nutrition consultation missed as a risk factor with a beta coefficient of 9.65 (95\% CI: $1.17-18.12 ; p=0.03)$.
\end{abstract}

Conclusions The increase in self-reported body weight is associated with a decrease in physical activity and the loss of face-toface visits to the Nutrition Unit.

Keywords Body weight · COVID-19 $\cdot$ Lockdown $\cdot$ Sleeve gastrectomy

\section{Introduction}

Since declaration of the COVID-19 pandemic on March 11, 2020, fast global spread of the disease has led to unprecedented measures. Coronavirus disease 2019 (COVID-19) is the infectious disease caused by the coronavirus SARS-CoV-2. COVID-19 produces a respiratory infection characterized by

Daniel de Luis

dadluis@yahoo.es

1 Endocrinology and Nutrition Research Center, School of Medicine, Department of Endocrinology and Nutrition, Hospital Clinico Universitario, University of Valladolid, C/Los perales 16 Simancas, 47130 Valladolid, Spain

2 Department of Surgery, Hospital Universitario Rio Hortega, Valladolid, Spain mild to severe symptoms. According to the National Center for Immunization and Respiratory Diseases, the high-risk categories for severe illness from COVID-19 are people aged 65 years and older, immunocompromised, and people with some chronic diseases such as obesity [1].

The early surge in cases threatened to overwhelm the National Health Systems and led people going into lockdown in order to "flatten the curve" of COVID-19 [2]. For example, one of the multiples implications is the abrupt cessation of outside activities for population in Spain, who by mandate had to remain in their homes during the "Lockdown." The resultant social isolation has led to unparalleled and precipitous changes in human behavior. There are reasons to be worried about housebound patients who have obesity, with a potential rapid weight gain. In lockdown, forced inactivity has coupled with altered eating habits often related with emotional troubles and stress $[3,4]$. This decrease of activity plus the 
increase in consumption of calorie-dense foods (snacking) and ultra-processed food is a factor strongly supported by observations in real-world settings [5]. Furthermore, the combination of online education, working from home, and social media usage has all caused screen time to surge [6]. All of these factors allow hypothesizing an increase in body weight during these weeks of lockdown that is likely to have metabolic repercussions in the near future and subsequently an increased risk of cardiovascular events.

During the COVID-19 pandemic, IFSO (International Federation for the Surgery of Obesity) recommended that all elective surgical and endoscopic cases for metabolic and bariatric surgery should be postponed. In addition, clinical and hospital visits are not recommended, too. Telemedicine online or phone follow-up has been proposed by this scientific society [7].

Due to the evolving nature of this pandemic, observational studies are important in order to gain more knowledge of the influence of this pandemic on the weight of patients with bariatric surgery. The aim of this cross-sectional study was to evaluate factors related to weight gain during lockdown in patients who have previously undergone a sleeve gastrectomy.

\section{Materials and Methods}

\section{Subjects and Clinical Investigation}

A group of 48 obese subjects with previous bariatric surgery has been consecutively enrolled from patients visiting the Department of Endocrinology and Nutrition for weight loss treatment and all participants have underwent robotic sleeve gastrectomy (SG). Robotic sleeve gastrectomy was performed using the DaVinci $\mathrm{X}^{\mathrm{TM}}$ (Intuitive Surgical Ltd, Oxford, UK) platform with 5 trocars (four 8-mm robotic trocars and one 12$\mathrm{mm}$ trocar for AirSeal ${ }^{\circledR}$ iFS (CONMED, NY, USA) insufflation device and stapling). The gastrocolic ligament was transected with the robotic Vessel Sealer Extend ${ }^{\mathrm{TM}}$ (Intuitive Surgical Ltd, Oxford, UK). The greater curvature was transected with the Signia ${ }^{\mathrm{TM}}$ Stapling System (Medtronic, Minneapolis, USA) and Endo GIA ${ }^{\mathrm{TM}}$ with Tri-Staple ${ }^{\mathrm{TM}}$ Technology (Medtronic, Minneapolis, USA), starting $6 \mathrm{~cm}$ from the pylorus to the angle of His. A 36-Fr bougie was used for calibration.

These 48 obese Caucasian subjects were enrolled in a nonprobabilistic consecutive method of sampling. After a 7-week confinement period from March 17 until May 8, a telephone survey was conducted. We recovered the anthropometric and biochemical information from the electronic medical record of the last visit to our Nutrition Unit. The recruited obese subjects fulfilled the following inclusion criteria: active monitoring in the Nutrition Unit of our hospital, currently a resident in our health area, and at least 18 months after the bariatric surgery (sleeve gastrectomy). The exclusion criteria were age under 18 years, severe renal or hepatic disorders, malignant tumor, or active COVID-19 disease during the study period. The Ethics Committee (HCUVA Committee) approved the study, and the study was in accordance with the guidelines laid down in the Declaration of Helsinki (PIP-GR20-1859). All participants have provided written informed consent.

In all participants, we recovered from their electronic medical record the next data of the last face-to-face visit and in the pre-surgical visit, systolic and diastolic blood pressure, and anthropometric parameters (weight, height, body mass index (BMI), and waist circumference). The next biochemical data were recovered from the medical record: glucose, insulin, total cholesterol, LDL-cholesterol, HDL-cholesterol, and triglycerides.

\section{Phone Questionnaire}

After the lockdown period of 7 weeks, a 15 -min telephone interview was conducted with the following questions: (1) Have you realized regular physical exercise? (yes/no); (2) How many minutes per week of exercise? (numerical); (3) What is your current weight? (numerical); (4) According to your perception, have you gained weight? (yes/no); (5) If the answer was "yes," how much weight have you gained? (numerical) (patient was recommended to weigh himself on his homologated home scale to obtain this result); (6) Do you take snacks between meals during the lockdown? (numerical); (7) How many meals do you eat per day since confinement? (numerical); (8) How many main meals did you eat before confinement? (numerical); (9) How many area $\left(\mathrm{m}^{2}\right)$ does your home have? (numerical); (10) How many members make up your family? (numerical); (11) Do you have a pet? (yes/no); (12) If you have been able to go outside with your pet, how many minutes per week? (numerical); (13) How many hours a day of TV did you watch before confinement? (numerical); (14) How many hours a day of TV do you watch now? (numerical).

Finally, the number of face-to-face visits to the Nutrition Unit that did not attend was recorded, as well as the number of visits realized to the Emergency Department during confinement. Patients who abandoned the intake of at least one vitamin or mineral supplement prescribed by their specialist and of any drug related to cardiovascular risk factors (blood hypertension, dyslipidemia, or diabetes mellitus) during 1 week were also recorded.

\section{Anthropometric and Biochemical Parameters from the Last Face-to-Face Visit}

In order to obtain the basal data of this population, the next parameters were recorded from electronic medical record (presurgical data and the last face-to-face visit in our Nutrition Unit); body weight was measured using scales and recorded to the nearest $50 \mathrm{~g}$. Height was measured with a tape measure. 
Body mass index (BMI) was calculated as body weight (in $\mathrm{kg}$ ) divided by height (in $\mathrm{m}^{2}$ ). Waist circumference (WC) was measured at the umbilical level. The next biochemical parameters were obtained from medical record; serum total cholesterol and triglyceride levels were determined by the enzymatic colorimetric assay, while HDL-cholesterol was measured in the supernatant after precipitation of other lipoproteins by enzymatic methods.

\section{Statistical Analysis}

All the data were analyzed using SPSS for Windows software package, version 23.0 (SPSS Inc., Chicago, IL, USA). The sample size was calculated to detect an increase in selfreported body weight gain during confinement of $3 \mathrm{~kg}$ with $90 \%$ power and $5 \%$ significance. Non-parametric variables were analyzed with the Mann-Whitney test and Wilcoxon test. Parametric test was analyzed with Student's $T$ test, ANOVA test, and Bonferroni post hoc test. Qualitative variables and the presence of comorbidities were analyzed with the chisquare test, with Yates' correction as necessary and Fisher's test as necessary. Pearson's test and Spearman's test were used to correlate numerical variables. Multiple regression analyses adjusted by age and gender were used to calculate the beta coefficient and $95 \%$ confidence interval (CI) to estimate the association of self-reported body weight gain with potential risk factors. A $p$ value under 0.05 was considered statistically significant.

\section{Results}

A sample of 48 subjects with sleeve gastrectomy (SG) was evaluated. The mean age was $45.3 \pm 8.0$ years (range: $23-61$ ) and the last mean body mass index (BMI) was $32.5 \pm 7.5 \mathrm{~kg} / \mathrm{m}^{2}$ (range: 28.6-34.2). Gender distribution was 38 females (79.2\%) and 10 males $(20.8 \%)$.

After a sleeve gastrectomy performed on average $41 \pm 12$ months, Table 1 reports biochemical and epidemiological data of the study population in the last face-to-face visit in the hospital and in the pre-surgical time. The next parameters showed significant statistical improvements between pre-surgical time and the last face-to-face visit data: body mass index (BMI) (delta: $5.7 \pm 0.9 \mathrm{~kg} / \mathrm{m}^{2} ; p=0.01$ ), weight (delta: $41.3 \pm 3.9 \mathrm{~kg} ; p=0.001$ ), waist circumference (delta: $23.1 \pm 3.4 \mathrm{~cm} ; p=0.02$ ), systolic blood pressure (delta: 15.2 $\pm 4.1 \mathrm{mmHg} ; p=0.01$ ), total cholesterol (delta: $35.1 \pm 9.2 \mathrm{mg} /$ $\mathrm{dl} ; p=0.03$ ), LDL-cholesterol (delta: $32.2 \pm 9.9 \mathrm{~kg} ; p=0.04)$, triglycerides (delta: $44.1 \pm 8.8 \mathrm{mg} / \mathrm{dl} ; p=0.02$ ), insulin levels (delta: $14.6 \pm 2.3 \mathrm{UI} / 1 ; p=0.01$ ), and HOMA-IR (delta: 3.0 $\pm 0.7 \mathrm{~kg} ; p=0.02$ ).

Table 2 shows the results of the answers to the phone questionnaire. A $64.4 \%$ of the patients acknowledged having gained weight. The increase in self-reported body weight was $3.8 \pm 2.1 \mathrm{~kg}$ during the 7 weeks of lockdown. The sample analyzed showed a high physical activity rate of $63.8 \%$. The patients who recognized that they did a lot of exercise had an average in minutes of physical activity higher than those who considered that they did not do enough exercise (no regular exercise vs regular exercise: $31.4 \pm 21.1$ vs $401.6 \pm 19.9 \mathrm{~min} /$ week; $p=0.01$ ). And the self-reported body weight gain was lower in the latter (no regular exercise vs regular exercise: 4.6 \pm 0.9 vs $1.1 \pm 0.3 \mathrm{~kg} ; p=0.02$ ).

Regarding eating habits, the patients recognized snacking in $29.2 \%$ of the sample. The patients who acknowledged performing snaking presented higher selfreported body weight gain (no snacking vs snacking: 2.5 $\pm 1.1 \mathrm{vs} 3.8 \pm 0.7 \mathrm{~kg} ; p=0.04$ ) than those who did not confirm it. The mean number of meals per day that the subjects took prior to lockdown was similar to that they carried out during lockdown (pre-lockdown: $4.6 \pm 0.8$ units vs during lockdown: $4.9 \pm 0.9$ meals; $p=0.43)$. Regarding the selfreported hours of TV per day, there was a significant increase in the hours during lockdown (TV hours prelockdown vs TV hours during lockdown: $2.45 \pm 0.7$ vs 3.65 $\pm 0.9 \mathrm{~h} ; p=0.01$ ). The presence of pets in the home was not related to self-reported body weight gain (no pet vs pet: 3.92 \pm 1.1 vs $3.48 \pm 1.2 \mathrm{~kg} ; p=0.29$ ).

The correlation analysis between the self-reported gained body weight of the patients and quantitative variables showed a negative correlation with minutes of exercise $(r=-0.405 ; p=0.01)$. The correlation analysis with the following parameters did not show statistically significant association with self-reported weight gain: "house area in $\mathrm{m}^{2}$ ", $(r=0.18 ; p=0.54)$, "number of family members at home" $(r=0.11 ; p=0.49)$, "minutes invested in the activity outside the home with the pet" $(r=0.17 ; p=0.51)$, and "number of hours on TV" $(r=0.28 ; p=0.12)$.

Finally, the number of face-to-face visits to the Nutrition Unit that did not attend was $0.61 \pm 0.81$ (range: $0-4$ ) per patient. A $49.3 \%$ of subjects lost at least one visit. Patients who missed at least one visit to the Nutrition Unit had a greater selfreported weight gain (no lost vs lost: $1.0 \pm 0.4$ vs $3.9 \pm 0.8 \mathrm{~kg}$; $p=0.04)$. The average number of visits to the Emergency Department during lockdown was $0.16 \pm 0.35$ (range: 0-2) per patient. A $17.6 \%$ of subjects visited the Emergency Department at least once. Only $3.4 \%$ of patients ( 2 patients) abandoned the intake of at least one vitamin or mineral supplement prescribed by their specialist and no patient stopped the drug therapy to blood hypertension.

In the multiple regression analysis with self-reported body weight gain as a dependent variable and adjusted for age and sex, the physical activity (minutes/week) remained as a protective factor with a beta coefficient of - 0.09 (95\% CI: 0.001 to $0.016 ; p=0.03$ ). The number of face-to-face appointments in the nutrition consultation remained as a risk factor 
Table 1 Epidemiological and biochemical parameters

\begin{tabular}{llll}
\hline $\begin{array}{l}\text { Parameters } \\
\text { Total group, } n=48\end{array}$ & Pre-surgical data & Last face-to-face visit & $p$ \\
\hline Age (years) & $40.3 \pm 6.1$ & $45.3 \pm 8.1$ & 0.03 \\
BMI & $38.2 \pm 7.1$ & $32.5 \pm 7.5$ & 0.01 \\
Weight (kg) & $128.7 \pm 11.3$ & $87.7 \pm 11.3$ & 0.001 \\
WC (cm) & $122.6 \pm 18.0$ & $99.6 \pm 14.0$ & 0.002 \\
SBP (mmHg) & $131.1 \pm 5.1$ & $116.1 \pm 4.1$ & 0.01 \\
DBP (mmHg) & $84.7 \pm 3.8$ & $83.7 \pm 3.8$ & 0.18 \\
Fasting glucose (mg/dl) & $109.1 \pm 7.9$ & $96.7 \pm 13.9$ & 0.01 \\
Total cholesterol (mg/dl) & $219.9 \pm 11.8$ & $185.9 \pm 12.8$ & 0.03 \\
LDL-cholesterol (mg/dl) & $144.1 \pm 30.9$ & $112.0 \pm 35.9$ & 0.04 \\
HDL-cholesterol (mg/dl) & $58.1 \pm 9.1$ & $61.7 \pm 12.1$ & 0.18 \\
Triglycerides (mg/dl) & $137.4 \pm 23.1$ & $93.4 \pm 43.1$ & 0.02 \\
Insulin (mUI/l) & $26.1 \pm 6.0$ & $11.9 \pm 7.0$ & 0.01 \\
HOMA-IR (units) & $6.3 \pm 1.1$ & $3.3 \pm 2.1$ & 0.02 \\
\hline
\end{tabular}

$B M I$ body mass index, $D B P$ diastolic blood pressure, $S B P$ systolic blood pressure, $W C$ waist circumference, $L D L$ cholesterol low-density lipoprotein cholesterol, $H D L$-cholesterol high-density lipoprotein cholesterol, HOMA-IR homeostasis model assessment. $p<0.05$, between genders with a beta coefficient of 9.65 (95\% CI: $1.17-18.12 ; p=0.03$ ) in the model.

\section{Discussion}

The main finding of this cross-sectional study was the fact that the lockdown indicated by the Spanish health authorities for a period of 7 weeks during the first epidemic wave has produced a significant increase in self-reported body weight gain in this sample of obese patients with sleeve gastrectomy. This increase of body weight was associated with a decrease in physical activity and the loss of face-to-face visits to the Nutrition Unit.

The World Health Organization considers noncommunicable diseases, such as obesity, a major risk factor for becoming seriously ill with the novel coronavirus $[8,9]$. For example, Lighter et al. [10] have demonstrated that obesity in patients younger than 60 years is a risk factor for COVID-19 hospital admission. Furthermore, some national series of patients indicates that almost $90 \%$ of deaths occurred in patients with non-communicable diseases, such as obesity, diabetes mellitus type 2, and hypertension [10]. For all the previously mentioned risk factors, obesity seems to be a risk
Table 2 Epidemiological questionnaire

\begin{tabular}{ll}
\hline Questions & Total group, $n=48$ \\
\hline Have you performed regular physical exercise? (yes/no) & $63.8 \% / 36.2 \%$ \\
How many minutes per week of physical exercise? (min) & $308.1 \pm 212.6$ \\
What is your current weight? (kg) & $90.9 \pm 18.7$ \\
According to your perception, have you gained weight? (yes/no) & $64.4 \% / 35.6 \%$ \\
How much weight have you gained? (kg) & $2.5 \pm 2.1$ \\
Do you take snacks between meals during the lockdown? (yes/no) & $29.2 \% / 70.8 \%$ \\
How many meals do you eat per day since confinement? & $4.7 \pm 0.9$ \\
How many main meals did you eat before confinement? & $4.6 \pm 0.8$ \\
How many m ${ }^{2}$ does your home have? & $109.1 \pm 34.9$ \\
How many members make up your family? & $2.7 \pm 1.1$ \\
Do you have a pet? (yes/no) & $31.2 \% / 68.8 \%$ \\
If you have been able to go outside with your pet, how many minutes per week? & $222.5 \pm 44.1$ \\
How many hours a day of TV did you watch before confinement? & $2.45 \pm 0.7$ \\
How many hours a day of TV do you watch now? & $3.65 \pm 0.9$
\end{tabular}


factor for poor adverse outcome of COVID-19 disease, and this propensity of subjects with obesity to develop more complications that are serious could be due to some factors such as the delayed and ineffective immune response and the chronic inflammatory status of these patients.

In this stage, outpatient visit of bariatric patients has been suspended or replaced by video consultations. Our health authorities did not allow face-to-face visits to certain services and pathologies during the first lockdown ( 7 weeks), and one of them was obese patients. The health authorities tried to prioritize the face-to-face visits to the external consultations of the hospital to certain serious pathologies, for example, cancer patients and patients with data of ischemic heart disease. For all this, we decided to make these telephone visits and to report the results. Traditional consultations consisted of 15-20-min face-to-face consultations with a prior blood test and face-to-face anthropometry of the patient; however, telephone visits involve questionnaires like the one used, without blood tests and with anthropometric data self-reported by the patients. Bariatric surgery has been inevitably affected by the COVID-19 pandemic, as indicated by Lazaridis et al. [11] in a worldwide survey. In our study, we have detected how a $49.3 \%$ of patients did not attend their visit in the Nutrition Unit and this was related to weight gain. On the other hand, limitations in mobility decreased the time of physical activity, which was also related to this body weight gain. The inability to go to work and exercise, emotional stress, and financial problems are providing the perfect milieu for the weight increase in subjects with bariatric surgery. Recently, He et al. [12] reported in an online survey in China that females with $\mathrm{BMI}>25 \mathrm{~kg} / \mathrm{m}^{2}$ gained weight during lockdown. In this study, the average steps per day and the average moderate- or vigorous-intensity exercise time declined. The changes in body weight inversely correlated with these changes in physical activity. Based on this survey, the authors considered that the effect of weight gain due to reduced physical activity was more powerful than the effect of calorie intake [12].

In our study, the self-reported body weight gain has not been related to dietary variables such as number of meals per day and snacking. The effect of COVID-19 lockdown on lifestyle in obese children has been demonstrated in some studies [13]. In this investigation [13], an increase in the time of sleep, a decrease in sports activities, and an increase in food intake such as fried potatoes, sugary drinks, and red meat were reported. However, the effect of lockdown and these changes in lifestyle habits on weight was not evaluated in this work. In our study, we did not report that snacking increases the risk of self-reported body weight gain in multivariant analysis. Perhaps during the lockdown, other psychological factors have been very important; it has been demonstrated that individuals under psychological stress consumed higher amounts of energy and dense snacks, particularly fatty and sweet snacks $[5,14,15]$. This worsening of the psychological variables has been demonstrated among patients with obesity during lockdown [16]. The relationship between nutritional intake and weight gain in patients with bariatric surgery during lockdown is an area of interest considering the aforementioned antecedents. However, the studies are scarce and partial. For example, Nicoletti et al. [17] have demonstrated an inappropriate nutritional intake in postoperative bariatric patients during the COVID-19 lockdown. They observed that almost $90 \%$ of the patients did not reach the necessary protein intake and $25 \%$ ate too many ultra-processed foods. However, in this work, the effect on body weight of these changes in nutritional intake was not evaluated. In other national survey with bariatric patients [18], 30\% of responders admitted having put on weight, significantly more in the group of preoperative patients $(43.8 \%)$ than postoperative patients $(22.7 \%)$. Only $20.9 \%$ of patients had a possibility of continuing direct bariatric care. However, in this study, neither the physical activity nor the weight that the patients gained during the lockdown period was collected. Finally, the majority of patients planned to increase their physical activity after the pandemic [18].

Furthermore, our interviewed subjects increased TV hours during confinement, although it was not directly related to the self-reported weight gain reported in our survey. Perhaps the high number of hours of TV that the subjects already reported before confinement has not allowed us to detect this influence [19].

Limitations of our study are, firstly, it is the relatively small sample size and short period of the study. Secondly, it is a cross-sectional study, so we cannot draw any causal conclusions. Thirdly, the body weight of the patients is self-reported, with under-estimation biases. Moreover, there is a generally strong agreement between self-reported body weight and clinic weights [20]. Fourthly, the time of exercise, snacking, and TV hours were also self-reported, with its corresponding biases. All these limitations are understandable given the exigencies operating during this pandemic. In addition, most of the patients have overpassed their nadir weight, so they should be in the "plateau" or slight weight regain phase after surgery, and the absence of a control group without lockdown does not allow ruling out this option, as an effect of the natural history of sleeve gastrectomy. Finally, we evaluated a cohort of patients with sleeve gastrectomy because it is the surgery that is being implanted in our public health system, by reducing hospital stay and comorbidities [21] and the data should be replicated with other types of bariatric, robotic, and non-robotic surgery.

The strength of the work is that these are real data from patients in our Nutrition Unit, which would allow us to take preventive measures to reduce weight gain in patients with bariatric surgery in successive epidemic waves.

In conclusion, the lockdown decreed during the pandemic by SARS-CoV-2 has produced an increase in self-reported body weight in obese subjects with sleeve gastrectomy. This 
increase is associated with a decrease in physical activity and the loss of face-to-face visits to the Nutrition Unit. These observations point to the important need for implementation of preventive tools during periods of lockdown. Such measures could be telemedicine lifestyle programs; endocrinologists can offer online guidance encouraging healthy family habits, implementing healthy foods, and exercise programs [22]. The lack of information regarding the increase risk of illness for subjects with morbid obesity might increase anxiety given that these subjects have now been categorized as vulnerable to severe illness if they contact COVID-19 [23]. However, we should encourage our bariatric patients in future pandemic waves to come to the clinic using all possible precautions of course.

Author Contribution Daniel Antonio de Luis designed the study and wrote the article. Olatz Izaola realized phone questionnaire. David Primo realized phone questionnaire. Beatriz Torres realized statistical analysis and designed the study. Emilia Gómez realized statistical analysis and designed the study. Juan José López Gómez designed the study and wrote the article. David Pacheco realized bariatric surgery.

\section{Declarations}

Ethical Approval All procedures performed in studies involving human participants were in accordance with the ethical standards of the institutional and/or national research committee (HVUVA committee PIPGR20-1859) and with the 1964 Helsinki Declaration and its later amendments or comparable ethical standards.

Informed Consent Informed consent was obtained from all individual participants included in the study.

Conflict of Interest The authors declare no competing interests.

\section{References}

1. Muscogiuri G, Pugliese G, Barrea L, et al. Commentary: Obesity: the "Achilles heel" for COVID-19? Metabolism. 2020;108:154251. https://doi.org/10.1016/j.metabol.2020.154251.

2. Khan MA, Moverley Smith JE. "Covibesity," a new pandemic. Obes Med. 2020;19:100282. https://doi.org/10.1016/j.obmed. 2020.100282.

3. Salgin B, Norris SA, Prentice P, et al. Even transient rapid infancy weight gain is associated with higher BMI in young adults and earlier menarche. Int J Obes. 2015;39(6):939-44.

4. Zouhal H, Ben Abderrahman A, Khodamoradi A, et al. Effects of physical training on anthropometrics, physical and physiological capacities in individuals with obesity: a systematic review. Obes Rev. 2020; https://doi.org/10.1111/obr.13039.

5. Camilleri GM, Caroline M, Kesse-guyot E, et al. Péneau. The associations between emotional eating and consumption of energydense snack foods are modified by sex and depressive symptomology. J Nutr. 2014;144:1264-73.
6. Nagata JM, Abdel Magid HS, Pettee GK. Screen time for children and adolescents during the coronavirus disease 2019 pandemic. Obesity (Silver Spring). 2020;28:1582-3.

7. Yang W, Wang C, Shikora S, et al. Recommendations for metabolic and bariatric surgery during the COVID-19 pandemic from IFSO. Obes Surg. 2020;30:2071-3.

8. Ryan DH, Ravussin E, Heymsfield S. COVID 19 and the patient with obesity - the editors speak out. Obesity (Silver Spring). 2020;28:847.

9. Lighter J, Phillips M, Hochman S, et al. Obesity in patients younger than 60 years is a risk factor for COVID-19 hospital admission. Clin Infect Dis. 2020;71:896-7.

10. Malavazos AE, Corsi Romanelli MM, Bandera F, et al. Targeting the adipose tissue in COVID-19. Obesity (Silver Spring). 2020;28: 1178-9.

11. Lazaridis II, Kraljević M, Schneider R, et al. The impact of the COVID-19 pandemic on bariatric surgery: results from a worldwide survey. Obes Surg. 2020;30:4428-36.

12. He M, Xian Y, Lv X, et al. Changes in body weight, physical activity, and lifestyle during the semi-lockdown period after the outbreak of COVID-19 in China: an online survey. Disaster Med Public Health Prep. 2020;14:1-6.

13. Pietrobelli A, Pecoraro L, Ferruzzi A, et al. Effects of COVID-19 lockdown on lifestyle behaviors in children with obesity living in Verona, Italy: a longitudinal study. Obesity (Silver Spring). 2020;28:1382-5.

14. Njike VY, Smith TM, Shuval O, et al. Snack food, satiety, and weight. Adv Nutr. 2016;7(5):866-78.

15. Larson N, Story M. A review of snacking patterns among children and adolescents: what are the implications of snacking for weight status? Child Obes. 2013;9:104-15.

16. Almandoz JP, Xie L, Schellinger JN, et al. Impact of COVID-19 stay-at-home orders on weight-related behaviours among patients with obesity. Clin Obes. 2020;10(5):e12386. https://doi.org/10. 1111/cob.12386.

17. Nicoletti CF, Esteves GP, Genario R, et al. Nutritional inadequacies among post-bariatric patients during COVID-19 quarantine in Sao Paulo, Brazil. Obes Surg. 2020;24:1-5.

18. Walędziak M, Różańska-Walędziak A, Pędziwiatr $M$, et al. Bariatric surgery during COVID-19 pandemic from patients' point of view-the results of a national survey. J Clin Med. 2020;9(6): 1697. https://doi.org/10.3390/jcm9061697.

19. Wansink B, Kim J. Bad popcorn in big buckets: portion size can influence intake as much as taste. J Nutr Educ Behav. 2005;37: 242-5.

20. Alberga AS, Edache Y, Forhan M, et al. Weight biass and health care utilization: a scoping review. Prim Health Care Res Dev. 2019;20:e116.

21. Scarritt T, Hsu CH, Maegawa FB, Ayala AE, Mobily M, Ghaderi I. Trends in utilization and perioperative outcomes in robotic-assisted bariatric surgery using the MBSAQIP database: a 4-year analysis. Obes Surg. 2020.

22. Woo Baidal JA, Chang J, Hulse E, et al. Zooming toward a telehealth solution for vulnerable children with obesity during coronavirus disease 2019. Obesity (Silver Spring). 2020;28:1184-6.

23. Flint SW, Tahrani AA. COVID-19 and obesity-lack of clarity, guidance, and implications for care. Lancet Diabetes Endocrinol. 2020;8:474-5.

Publisher's Note Springer Nature remains neutral with regard to jurisdictional claims in published maps and institutional affiliations. 